\title{
MODELING AND SIGNAL PROCESSING OF ACOUSTIC GUNSHOT RECORDINGS
}

\author{
Robert C. Maher \\ Department of Electrical and Computer Engineering, Montana State University, Bozeman MT 59717 \\ rob.maher@montana.edu
}

\begin{abstract}
Audio recordings of gunshots can provide information about the gun location with respect to the microphone(s), the speed and trajectory of the projectile, and in some cases the type of firearm and ammunition. Recordings obtained under carefully controlled conditions can be well-modeled by geometrical acoustics. Special acoustic processing systems for real time gunshot detection and localization are used by the military and law enforcement agencies for sniper detection. Forensic analysis of audio recordings is also used to provide evidence in criminal and civil cases. This paper reviews the distinctive features and limitations of acoustic gunshot analysis using DSP techniques.
\end{abstract}

Index Terms - shock wave acoustics, gunshots

\section{INTRODUCTION}

A conventional firearm uses a confined explosive charge to propel the bullet out of the gun barrel. The hot, rapidly expanding gases cause an acoustic muzzle blast to emerge from the barrel. This acoustic disturbance lasts 3-5 milliseconds and propagates through the air at the speed of sound $(c)$.
The muzzle blast is not necessarily a reliable acoustic source for analysis. For most firearms the sound level of the muzzle blast is strongest in the direction the barrel is pointing, and decreases as the off-axis angle increases. The blast may also be obscured by barriers and other obstacles blocking the direct path between the firearm and the microphone location. Furthermore, some firearms can be equipped with an acoustical suppressor ("silencer") to alter or reduce the muzzle blast sound level $[1,2,4]$.

A second source of acoustic information is present if the bullet travels at supersonic speed, $V>c$. The supersonic projectile causes an acoustic shock wave that propagates away from the bullet's path. The shock wave expands as a cone behind the bullet, with the wave front propagating outward at the speed of sound. The shock wave cone has an inner angle, $\theta_{M}=\arcsin (1 / M)$, where $M=V / c$ is the Mach Number. $\theta_{M}$ is referred to as the Mach angle. The shock wave geometry is shown in Fig. 1.

With a very fast bullet, $M$ is large and $\theta_{M}$ becomes small, causing the shock wave to propagate nearly perpendicularly to the bullet's trajectory. For example, a bullet traveling at 3000 feet per second at room temperature has $M=2.67$, giving $\theta_{M}=\sim 22^{\circ}$. On the other hand, if the bullet is only slightly faster than the speed of sound, $M$ is approximately unity, $\theta_{M}$ is nearly $90^{\circ}$, and the shock wave propagates

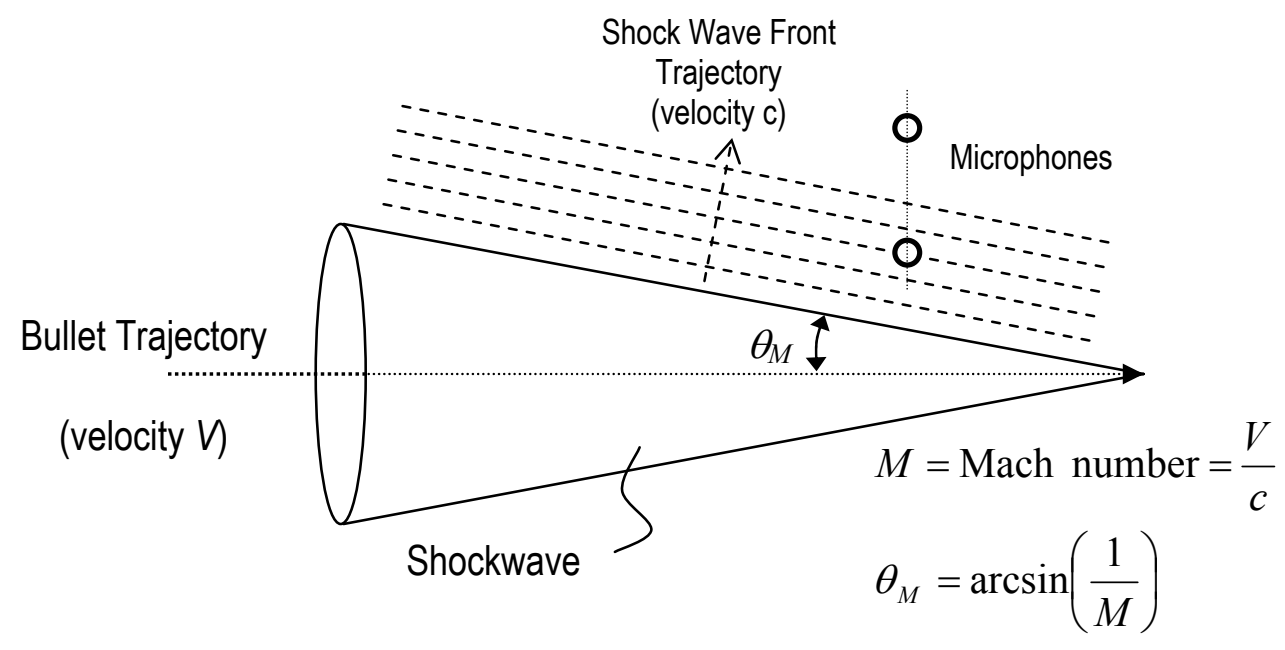

Figure 1: Supersonic bullet shock wave description 
nearly parallel to the bullet's path.

If two or more microphones are located at known locations within the path of the shock wave, the time of arrival difference(s) can be used to estimate the shock's propagation direction. Note, however, that determining the bullet's trajectory from the shock propagation vector requires knowledge of the bullet velocity, $V$. If $V$ is not known, then $M$ and $\theta_{M}$ are also not known, and the bullet's trajectory cannot be determined exactly without additional spatial information, as considered in Section 3 below.

The acoustic shock wave from the bullet has a very rapid rise to a positive overpressure maximum, followed by a corresponding under-pressure minimum. As the shock wave propagates the nonlinear behavior of the air causes the pressure disturbance to form an "N" shape with a rapid onset, a ramp to the minimum pressure, and then an abrupt offset. The time interval, $T$, of the "N" wave between the maximum over- and under-pressure instants is proportional to the size of the projectile. An expression for the "N" wave time interval is given by [3]:

$$
T \approx 1.82\left(\frac{d}{c}\right)\left(\frac{M x}{l}\right)^{\frac{1}{4}},
$$

where $d$ is the bullet diameter, $l$ is the bullet length, $c$ is the speed of sound, $M$ is the Mach number, and $x$ is the distance between the bullet's trajectory and the microphone at the point of closest approach (perpendicular distance between the bullet's path and the microphone). A typical bullet a few centimeters long has an intershock interval of less than $200 \mu \mathrm{sec}$, as shown in Fig. 2.

If solid surfaces are present nearby, the passing shock wave cone will be partially absorbed and partially reflected by the surface. Thus, a microphone in the vicinity will pick up both the original shock wave and the reflected shock

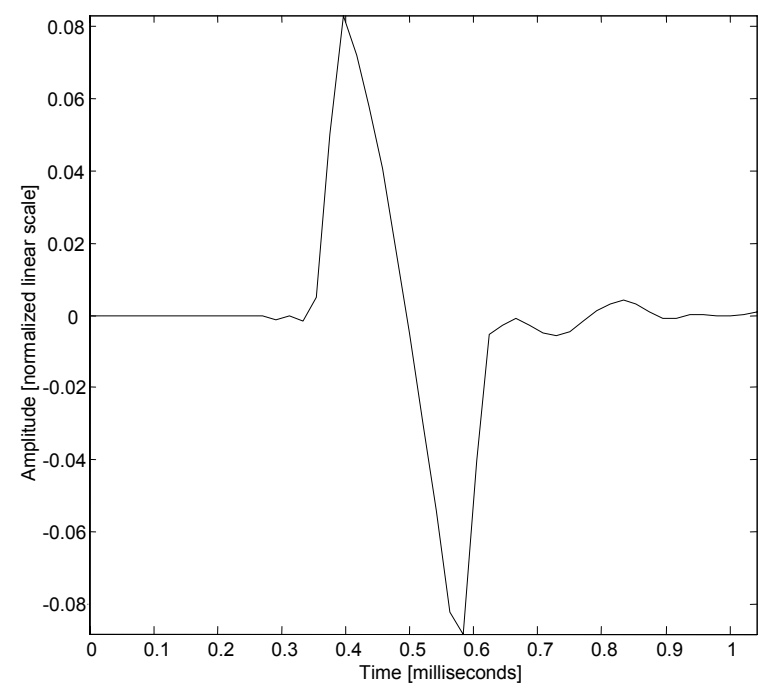

Figure 2: Shock wave recording ("N" wave)

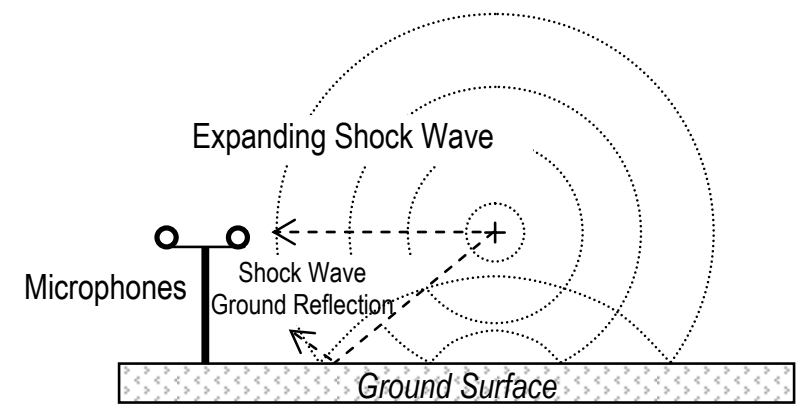

Figure 3: Shock wave ground reflection (elevation view, no scale, bullet into page)

wave with a delay corresponding to the path length difference. The ground reflection is depicted in Fig. 3.

If a supersonic projectile's journey from the firearm to the target is more than a few tens of meters, the effects of drag-induced deceleration may need to be taken into account $[4,5]$. The bullet is slowed by friction with the air and due to the conversion of its kinetic energy into the acoustic shock wave. As the projectile's speed decreases downrange, the shock wave Mach angle $\left(\theta_{M}\right)$ increases from its initial value where the bullet leaves the muzzle toward $90^{\circ}$, before the shock wave vanishes as the bullet slows to below the speed of sound. Thus, the actual shock wave boundary is convex rather than straight, as depicted in Fig. 4.

\section{MODELING AND MEASUREMENT EXAMPLE}

A Winchester 308 rifle was fired horizontally in a direction perpendicular to the plane of two omnidirectional microphones mounted one foot apart ( 0.305 meters) and 1.6 meters above the ground. The rifle was fired from shoulder

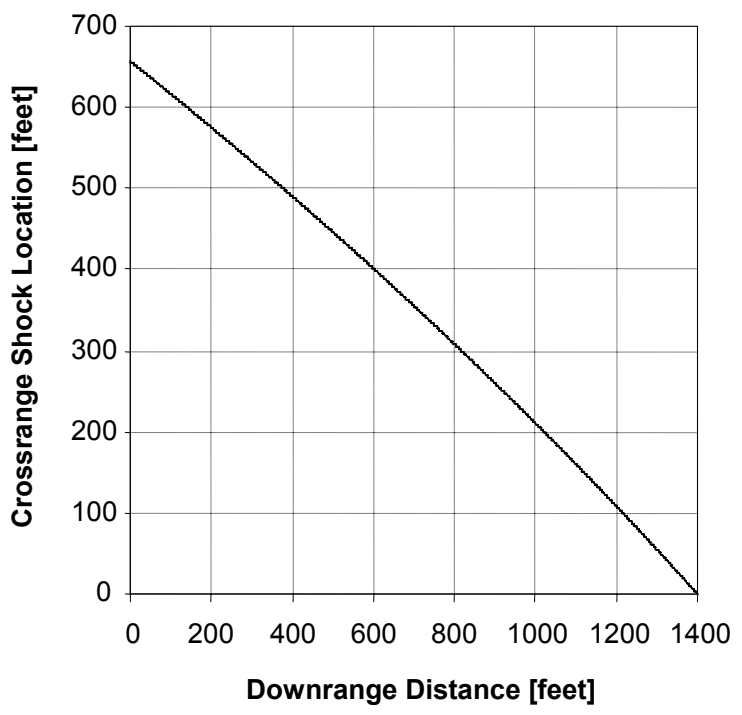

Figure 4: Shock wave front for NATO FNM 83-23 bullet (2750 ft/sec at muzzle, $1980 \mathrm{ft} / \mathrm{sec}$ at 1400 feet downrange) 
height (approximately 1.6 meters above the ground) at a distance of 6.3 meters from the plane of the microphones. The bullet crossed the microphone plane 3.7 meters from the nearest ("left") microphone. The bullet speed $(V)$ for the particular ammunition used was $2728 \mathrm{ft} / \mathrm{sec}(831.5 \mathrm{~m} / \mathrm{sec})$ and the speed of sound $(c)$ was $1075 \mathrm{ft} / \mathrm{sec}(328 \mathrm{~m} / \mathrm{sec})$ at approximately $20^{\circ} \mathrm{F}\left(-7^{\circ} \mathrm{C}\right)$. The resulting Mach number $(V / c)$ was 2.54 , giving a Mach cone angle $\left(\theta_{M}\right)$ of $23.2^{\circ}$. The geometry is shown in Fig. 5.

The predicted acoustic result within a few meters of the rifle begins with the bullet speeding away from the muzzle along path $\mathrm{A}$ at more than 2.5 times the speed of sound, trailing its $23.2^{\circ}$ shock wave cone. The shock wave front itself, expanding outward from the bullet's path at the speed of sound, will propagate away from the bullet's course in a direction parallel to paths B and B'.

The shock wave ray that reaches the microphone is launched when the bullet reaches position $X$. Thus, the total time between the gunshot and the shock wave arrival at the microphone consists of the bullet's time-of-flight at velocity $V$ from the muzzle to point $X$, plus the ensuing shock wave ray propagation at the speed of sound along ray path $B$. Geometrically, this total time is equal to the propagation time at the speed of sound along path B'. The ground reflection of the shock wave ray will propagate at the same azimuth as path B, but along the longer path from the muzzle to the ground and back up to the microphones.

The sound of the muzzle blast itself will travel directly from the gun to the microphones along path $\mathrm{C}$. The ground reflection of the muzzle blast arrives later due to the longer propagation distance from the muzzle to the ground and then back up to the microphones.

The predicted propagation times for the shock wave and

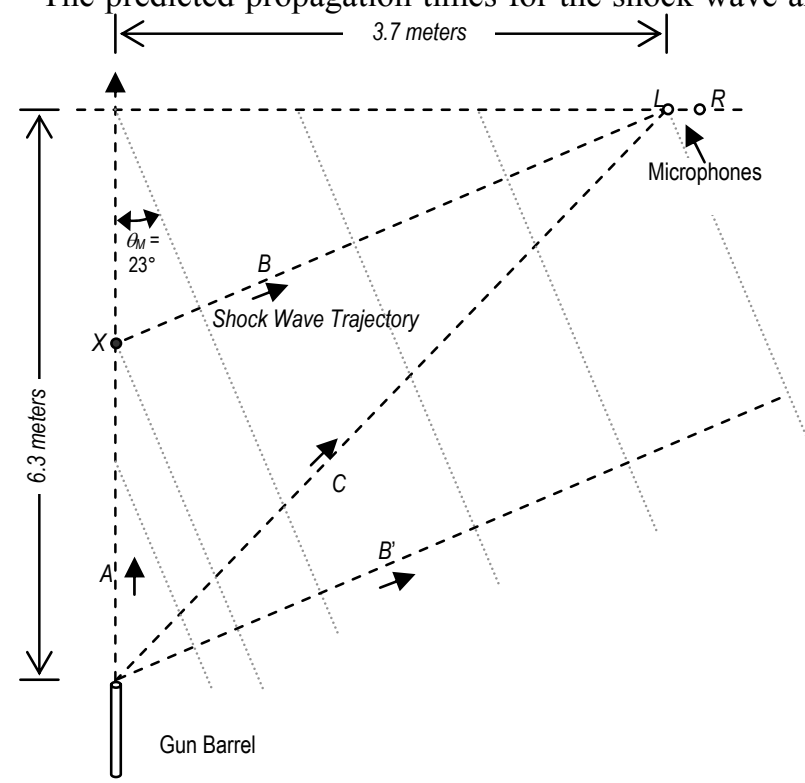

Figure 5: Test recording geometry (not to scale) muzzle blast arrivals at the microphones are shown in Table 1.

The two-channel audio recording obtained from this configuration is shown in Fig. 6. Using the arrival of the initial shock wave as the time reference, the measured time intervals and the percent discrepancy between the measured and predicted values are shown in Table 2 . The microphone signals were digitized with a $48 \mathrm{kHz}$ sample rate per channel so the timing accuracy is limited to 20.8 microseconds (1/48000), but the agreement between the predictions and the measurement are very good, which validates the geometrical acoustics model.

\section{TABLE 1: THEORETICAL LENGTHS AND TIMING}

\begin{tabular}{|l|c|c|}
\hline Path & Length & Time \\
\hline Bullet: muzzle to $X$ & $4.75 \mathrm{~m}$ & $5.71 \mathrm{~ms}$ \\
\hline Shock: path B (X to mic $L$ ) & $4.35 \mathrm{~m}$ & $12.3 \mathrm{~ms}$ \\
\hline Shock: path $B^{\prime}$ & $5.91 \mathrm{~m}$ & $18.0 \mathrm{~ms}$ \\
\hline Shock: ground refl. to $L$ & $6.63 \mathrm{~m}$ & $20.2 \mathrm{~ms}$ \\
\hline Shock: mic. $L$ to $R$ & $0.280 \mathrm{~m}$ & $0.855 \mathrm{~ms}$ \\
\hline Blast: path $C$ (muzzle to $L$ ) & $7.35 \mathrm{~m}$ & $22.4 \mathrm{~ms}$ \\
\hline Blast: ground refl. to $L$ & $7.93 \mathrm{~m}$ & $24.2 \mathrm{~ms}$ \\
\hline Blast: mic. L to R & $0.154 \mathrm{~m}$ & $0.470 \mathrm{~ms}$ \\
\hline
\end{tabular}

TABLE 2: MEASURED ACOUSTIC TIMING

\begin{tabular}{|l|c|c|}
\hline Interval & Measured & Discrepancy \\
\hline (1) Shock: refl. rel. arrival & $2.23 \mathrm{~ms}$ & $-0.037 \mathrm{~ms} \mathrm{(-1.7 \% )}$ \\
\hline (2) Shock: mic. $L$ to $R$ & $0.833 \mathrm{~ms}$ & $0.022 \mathrm{~ms}(2.6 \%)$ \\
\hline (3) Blast arrival rel. to Shock & $4.39 \mathrm{~ms}$ & $0.017 \mathrm{~ms}(0.38 \%)$ \\
\hline (4) Blast: refl. rel. arrival & $1.80 \mathrm{~ms}$ & $0.069 \mathrm{~ms} \mathrm{(3.8 \% )}$ \\
\hline (5) Blast: mic. L to R & $0.479 \mathrm{~ms}$ & $-0.005 \mathrm{~ms} \mathrm{(-1 \% )}$ \\
\hline
\end{tabular}

\section{GUNSHOT SOUND ANALYSIS}

In the preceding example the key geometric and ballistic variables were all assumed to be known. Although this allows a useful comparison and validation between the analytical and empirical results, it does not represent the more interesting inverse problem in which only the acoustical recording is measured and the geometrical and ballistic variables are to be determined. This is the realistic situation for sniper detection and localization systems, and also for most audio forensic gunshot recordings[1, 2, 3, 4].

\subsection{Number of microphones}

A typical acoustic detection problem involves estimating the bullet's shock wave trajectory, then deriving the azimuth, elevation, and (perhaps) distance of the shooter with respect to a reference point. A single microphone at a known 
location can determine the relative arrival time of the projectile's shock wave, the muzzle blast, and the ensuing acoustical reflections. Two microphones can estimate the elevation and distance of the bullet trajectory with respect to the known microphone locations, but the orientation of the trajectory and the bullet's speed are still ambiguous. Thus, in general, a set of at least four spatially distributed microphones are necessary [4]. Moreover, if the projectile's drag is to be included, the spatial distribution of the microphones must be sufficient to estimate the projectile's position-dependent Mach number so that the shock wave curvature can be estimated (cf. Fig. 4).

\subsection{Acoustical issues}

Persistent lore to the contrary, conventional audio recordings have not been reliable for identifying particular firearms $[1,2]$. Two gunshot recordings obtained from the same firearm may differ substantially unless the microphone is at a fixed location with respect to the firearm and the acoustical surroundings are identical. Especially if there is no direct acoustic path between the muzzle and the microphone, the received signal will be characterized by reflections and diffraction of the gunshot sound by nearby surfaces and obstacles. In this situation the audio recording will likely give more information about the acoustic surroundings (i.e., the acoustic impulse response) rather than the firearm or the projectile characteristics. Deconvolving the gunshot from the reflected sound and the reverberant clutter is generally a challenging problem $[1,2]$.

The muzzle blast sound is highly directional. Fig. 7 shows the acoustic recording obtained when the bullet's trajectory passes very close to the microphones, causing the shock wave reflections and the muzzle blast to arrive essentially simultaneously in both channels at the microphones. Fig. 8 shows the acoustic recording when the muzzle is facing away from the microphones: no shock wave is detected and the peak muzzle blast sound level is reduced by $20 \mathrm{~dB}$ or more compared to the direct shot in Fig. 7.

At distances far from the bullet's trajectory, the shock wave will have expanded sufficiently by spatial spreading that it may no longer be detectable compared to ambient noise. The non-linear propagation behavior of the shock wave will gradually lose its characteristic "N" wave shape and become a less abrupt " $\mathrm{S}$ " shaped pressure disturbance due to nonlinear dispersion [5].

A subsonic rifle or handgun produces a muzzle blast signal, but the subsonic bullet itself does not produce significant sound as it propagates through the air. An example recording of a handgun and subsonic bullet (HK USP compact, 40 Smith and Wesson, Federal Hydroshock)

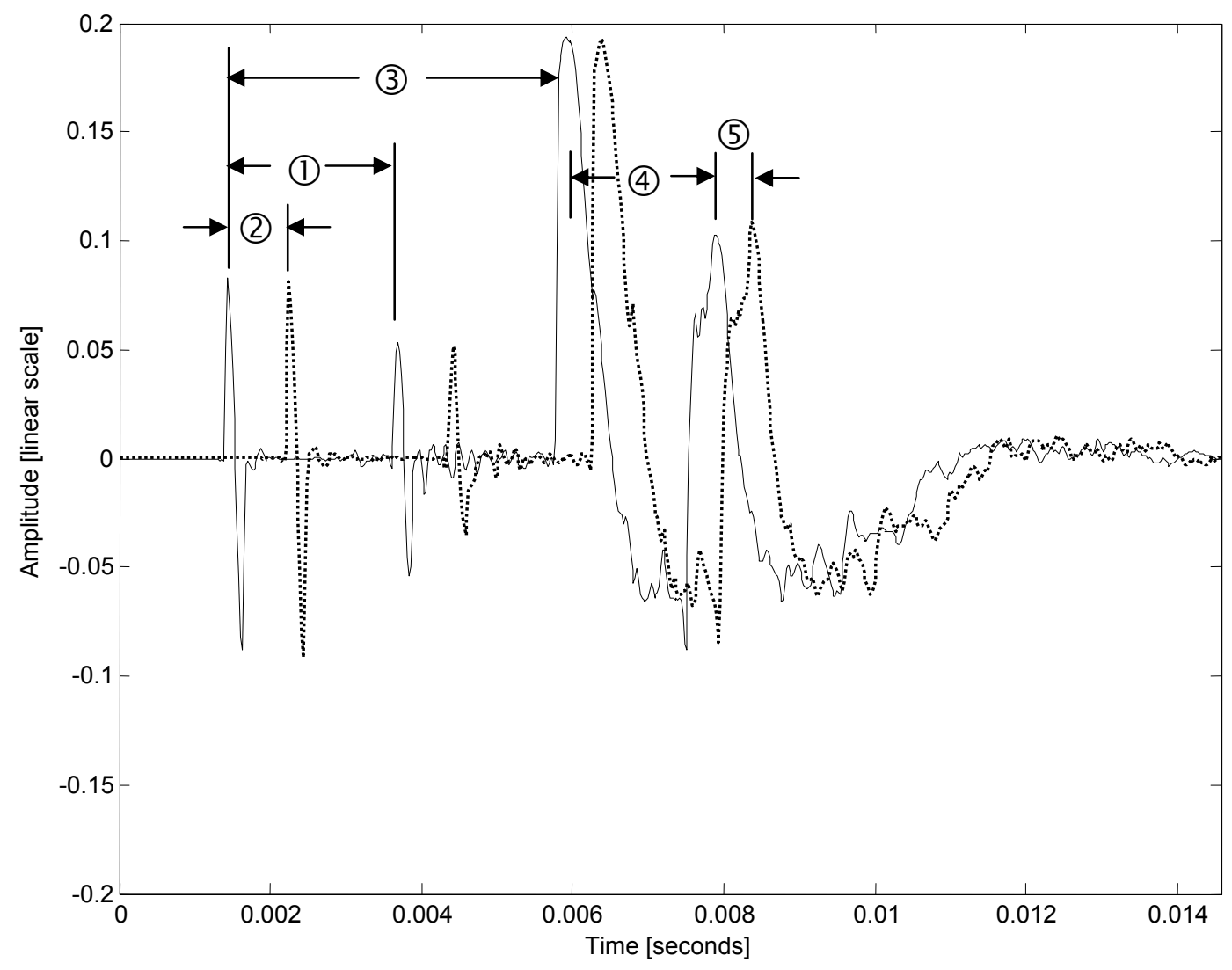

Figure 6: Two-channel audio recording (solid=left, dash=right) 


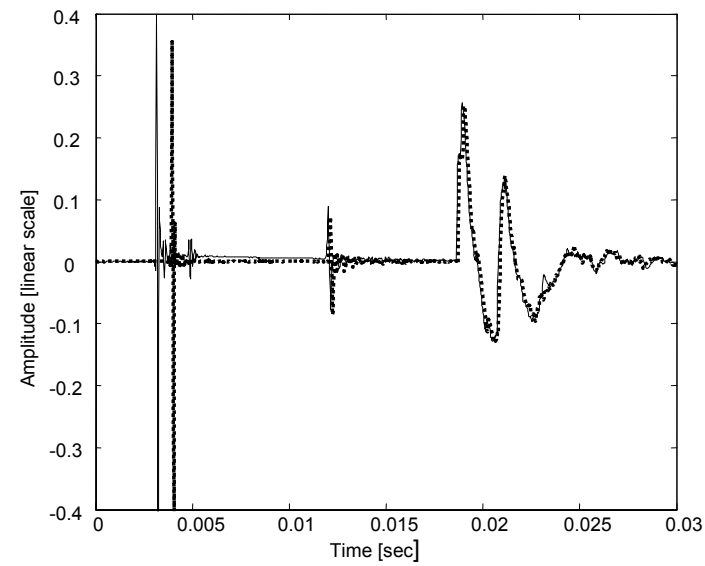

Figure 7: Gunshot recording, bullet trajectory passing close to microphones (solid=left, dash=right)

corresponding to the gun and microphone geometry of Fig. 5 is shown here in Fig. 9. Comparing the handgun recording in Fig. 9 to the previously described rifle recording of Fig. 6, no shock wave signature is apparent, and the muzzle blast is quieter and shorter in duration than the rifle shot.

\section{GUNSHOT PROCESSING CONSIDERATIONS}

The various acoustical characteristics described in the preceding section lead to several important recommendations for gunshot acoustical signal analysis.

- Reliable detection of shock waves usually involves identifying the abrupt "N" wave acoustic signature.

- Shock wave rise times are less than $2 \mu \mathrm{sec}$, so typical $48 \mathrm{kHz}$ audio sample rates and recording bandwidths are inadequate to fully characterize shock wave details [6]. Nevertheless, relative shock wave arrival times can still be deduced using multiple sensor channels at audio sample rates.

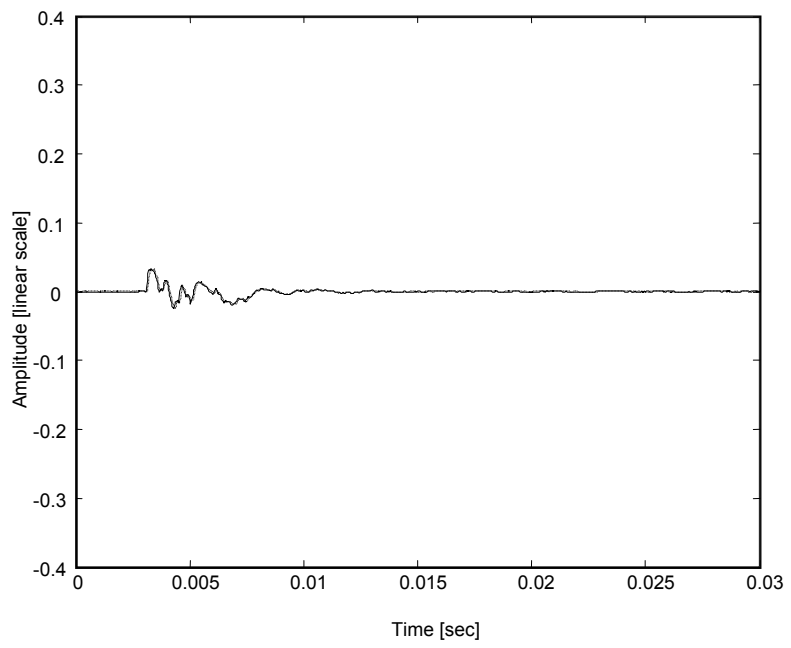

Figure 8: Gun shot recording with microphones behind the rifle (left and right channels coincident)
- Microphones located close to the firearm or within a few meters of a supersonic bullet's trajectory must handle peak sound pressure levels in excess of $160 \mathrm{~dB}$ re $20 \mu \mathrm{Pa}$ [6].

- If the muzzle is pointed away from the microphones at an angle such that the projectile's shock wave cone does not reach the microphones, only the muzzle blast will be observed.

\section{REFERENCES}

[1] Freytag, J.C., and Brustad, B.M., "A survey of audio forensic gunshot investigations," Proc. AES $12^{\text {th }}$ International Conf., Audio Forensics in the Digital Age, pp. 131-134, July 2005.

[2] Koenig, B.E., Hoffman, S.M., Nakasone, H., and Beck, S.D. "Signal convolution of recorded free-field gunshot sounds," J. Audio Eng. Soc., vol. 46(7/8), pp. 634-653, July/Aug 1998.

[3] Sadler, B.M., Pham, T., and Sadler, L.C., "Optimal and wavelet-based shock wave detection and estimation," J. Acoust. Soc. Am., vol 104(2), pt. 1, pp. 955-963. August 1998.

[4] Duckworth, G.L., Gilbert, D.C., and Barger, J.E., "Acoustic counter-sniper system," Proc. of SPIE, Vol. 2938, Command, Control, Communications, and Intelligence Systems for Law Enforcement, E.M. Carapezza and D. Spector, Eds., pp. 262-275, February 1997.

[5] Stoughton, R., "Measurements of small-caliber ballistic shock waves in air," J. Acoust. Soc. Am., vol. 102(2), pt. 1, pp. 781-787, August 1997.

[6] Weissler, P.G., and Kobal, M.T., "Noise of police firearms," J. Acoust. Soc. Am., vol. 56(5), pp. 1515-1522, November 1974.

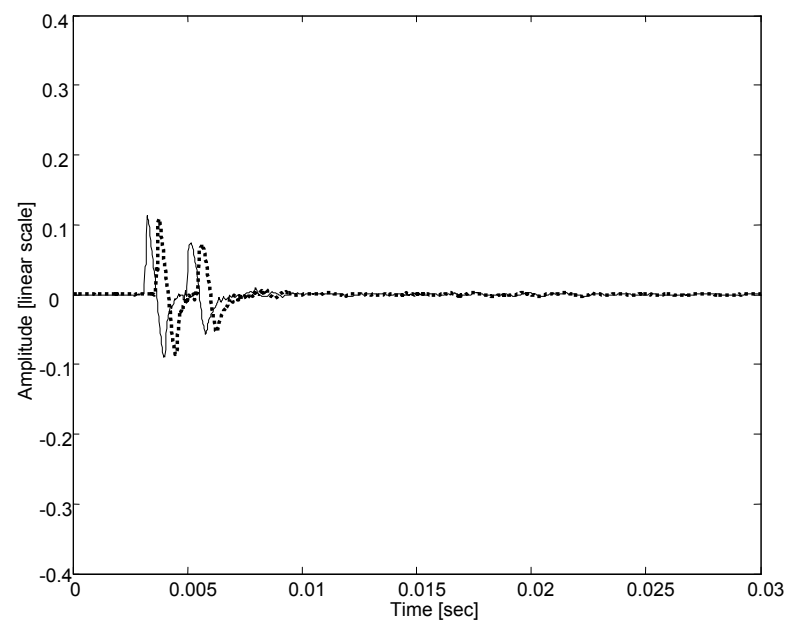

Figure 9: Handgun recording, sub-sonic bullet, same mic position as Figs. 5 and 6 (solid=left, dash=right) 